\title{
Volumetric MRI of the Lungs during Forced Expiration
}

\author{
Benjamin Paul Berman ${ }^{1}$, Abhishek Pandey ${ }^{2}$, Zhitao Li $^{2}$, Lindsie Jeffries ${ }^{3}$, Theodore \\ Trouard $^{3,4}$, Isabel Oliva ${ }^{4}$, Felipe Cortopassi ${ }^{4}$, Diego R. Martin ${ }^{4}$, Maria I. Altbach ${ }^{4}$, and Ali \\ $\operatorname{Bilgin}^{2,3,4}$ \\ ${ }^{1}$ Program in Applied Mathematics, University of Arizona, Tucson, Arizona, USA \\ ${ }^{2}$ Department of Electrical \& Computer Engineering, University of Arizona, Tucson, \\ Arizona, USA \\ ${ }^{3}$ Department of Biomedical Engineering, University of Arizona, Tucson, Arizona, USA \\ ${ }^{4}$ Department of Medical Imaging, University of Arizona, Tucson, Arizona, USA
}

This paper has been published by the journal Magnetic Resonance in Medicine. doi: $10.1002 / \mathrm{mrm} .25798$

The figures are found on pages $11-16$. 


\begin{abstract}
Purpose

Lung function is typically characterized by spirometer measurements, which do not offer spatially specific information. Imaging during exhalation provides spatial information but is challenging due to large movement over a short time. The purpose of this work is to provide a solution to lung imaging during forced expiration using accelerated MRI. The method uses radial golden angle stack-of-stars gradient echo acquisition and compressed sensing reconstruction.
\end{abstract}

\title{
Methods
}

A technique for dynamic 3D imaging of the lungs from highly undersampled data is developed and tested on six subjects. This method takes advantage of image sparsity, both spatially and temporally, including the use of reference frames called bookends. Sparsity, with respect to total variation, and residual from the bookends, enables reconstruction from an extremely limited amount of data.

\section{Results}

Dynamic 3D images can be captured at sub-150 ms temporal resolution, using only three (or less) acquired radial lines per slice per timepoint. The images have a spatial resolution of $4.6 \times 4.6 \times 10 \mathrm{~mm}$. Lung volume calculations based on image segmentation are compared to those from simultaneously acquired spirometer measurements.

\section{Conclusion}

Dynamic lung imaging during forced expiration is made possible by compressed sensing accelerated dynamic $3 \mathrm{D}$ radial MRI.

\section{Keywords}

spirometry, forced expiration, pulmonary, compressed sensing 


\section{Introduction}

Lung spirometry, which measures changes in lung volume as a function of time, additively from both lungs, is used to characterize lung diseases such as asthma, chronic obstructive pulmonary disease (COPD), and interstitial lung disease (ILD). Computed tomography (CT) and chest radiography (CXR) can be used to observe emphysema and hyperinflation $(1,2)$, but are not routinely used to characterize dynamic air flow and lung function. Instead, a spirometer can be used as part of a pulmonary function test (PFT) to measure the air flow from the lungs and airways during forced expiration to derive the forced expiratory volume (FEV) and the percentage of the FEV expired in the first second of effort $\left(\mathrm{FEV}_{1}\right)$.

The $\mathrm{FEV}_{1}$ is determined by having the patient inspire as deeply as possible and then expire maximally and as forcibly as possible. The forced vital capacity (FVC) represents the total expired volume during this maneuver. A normal subject has an $\mathrm{FEV}_{1}$ greater than $80 \%$ of the FVC.

PFTs can be used to assess diseases affecting biomechanical properties of the lungs and/or airways. However, PFT's may not be sensitive to early stages of most diseases. This is partly due to the lack of capacity for evaluating either lung separately, or regionally. It is known that pathological changes may only affect a small portion of the lungs (3), which makes spirometry potentially ineffective in early diagnosis. More sensitive methodology may lead to more timely treatment, improved prognosis, and better evaluation and regulation of therapy.

Magnetic resonance imaging (MRI) has been used in previous studies of pulmonary function and airflow. Imaging sequences need to be fast in order to account for respiratory motion, whether free breathing, paced breathing, or forced breathing. It has been most common to use dynamic 2D imaging methods, which are able to acquire fully sampled data at sufficient temporal resolution. Chen et al. (4) and Voorhees et al. (5) used grid-tagging in order to quantify lung motion during short breath maneuvers and paced breathing respectively; total imaging times per single image were approximately $150 \mathrm{~ms}$. Sundaram and Gee (6) examined regional biomechanics between respiratory phases using image registration, differentiating between healthy and diseased states in mice. Zhang et al. (7) implemented a tissue tracking method to study regional differences between healthy and asthmatic human lungs.

Methods involving hyperpolarized gas $\left({ }^{3} \mathrm{He}\right.$ or $\left.{ }^{129} \mathrm{Xe}\right)$ (8) have also been used for imaging the lungs. However, these methods contribute to costs and are focused on imaging the airways rather than lung biomechanics.

Since 2001 there have been many advances in lung imaging based on the volume and boundaries. In 2007, Eichinger et al. (9) commented that volumetric dynamic 3D (4D) lung images with high temporal and spatial resolution would be "the ideal step forward in real-time assessment of lung function." To circumvent the demanding time requirements of dynamic 3D acquisition, Plathow et al. (10) extrapolated lung volume using a collection of dynamic 2D images and the Barnhard and 
Lloyd elliptical model (11).

Achieving sufficient temporal resolution relies on accelerated imaging techniques such as parallel imaging and compressed sensing (CS). Tokuda et al. (12) used a 128-receiver coil and parallel imaging methods and achieved 3D image frames every $1.31 \mathrm{~s}$. CS was used for static 3D lung imaging with hyperpolarized Helium by Ajraoui et al. (13); they reported acceleration factors of up to 4 using spatial sparse transformations. Most recently, Lingala et al. (14) used CS methods for dynamic 3D imaging of the lungs during free breathing with spatial and temporal resolutions of $2.37 \times 2.37 \times 4 \mathrm{~mm}$ and $720 \mathrm{~ms}$ per frame. These images captured natural respiration with nearly complete coverage of the lungs.

Because the $\mathrm{FEV}_{1}$ is recorded over one second, the temporal resolution of the 3D image frames needs to be high $(<200 \mathrm{~ms})$ in order to provide sufficient time-point measurements. We present here an imaging protocol capable of dynamic 3D lung imaging at unprecedented temporal resolution, as fine as $50 \mathrm{~ms}$ per frame - sufficiently adequate for tests such as $\mathrm{FEV}_{1}$.

\section{Methods}

\section{MRI Acquisition}

This study was performed in compliance with the human research subjects guidelines, and the institutional review board. Six healthy subjects with no history of lung disease were recruited on a voluntary basis.

Imaging was performed on a 1.5T MRI scanner (Aera, Siemens Healthcare, Malvern, PA, USA) using a radial golden angle stack-of-stars (15) gradient echo pulse sequence. Data were received from 30 coils covering the chest and spine using the standard RF coils in the scanner. The 18-element flexible body coil was used to cover the anterior section; 12 elements from the spine matrix coil (which is integrated into the patient table) were used to cover the posterior section. Acquisition parameters were: $\mathrm{TR}=2.0 \mathrm{~ms}$; $\mathrm{TE}=0.77 \mathrm{~ms}$; flip angle $\alpha=1^{\circ}$; and receiver bandwidth $=1565$ $\mathrm{Hz} /$ pixel. The 3D cylindrical stack-of-stars trajectory had coronal orientation with saturation bands placed above and below the lungs to reduce signal from anatomy outside the field-of-view (FOV). Data were acquired with 96 readout points, $10 \mathrm{~mm}$ slice thickness and 22-26 slices based upon subject size to achieve coverage of the entire lungs. These parameters led to an image matrix of $96 \times 96 \times 22-26$ voxels, and a resolution of $4.38-4.69 \times 4.38-4.69 \times 10 \mathrm{~mm}$.

Study participants were instructed through a headset to perform a forced expiration maneuver within the scanner. The maneuver had three phases:

1. Initial bookend - Inhale deeply and hold a maximum inspiration for $5 \mathrm{~s}$

2. Dynamic phase - Forcibly expire with a maximum effort

3. Final bookend - Maintain exhalation effort for $5 \mathrm{~s}$ 
Data were acquired for the full experiment using continuous scanning, with a total scan time between 20 to $30 \mathrm{sec}$ which includes the initial inhalation and the final free breathing periods (see Figure 1).

The radial golden angle stack-of-stars cylindrical k-space view ordering enables temporal flexibility. The radial readouts are acquired in each slice before the angle is incremented by $\pi / \phi \mathrm{rad}$ $\left(\approx 111.25^{\circ}\right)$, which defines the golden angle, where $\phi=(1+\sqrt{5}) / 2$. Regardless of how the data are binned into dynamic frames, the golden angle ensures no overlap and nearly uniform coverage (16). A through-slice vector of data with $\left(k_{x}, k_{y}\right)=(0,0)$ is measured with each angle, and the correlation of these vectors - with respect to a reference vector from the same data - clearly exhibits the forced breathing maneuver, as shown in Figure 1.

The use of $\alpha=1^{\circ}$ leads to a proton density weighted image. The flat contrast of the image will lead to lower the total variation (TV) (Eqs. [1] to [4]), a reconstruction regularizer that relies on a piecewise-constant image. Because we are only concerned with the boundary of the lung for the purpose of total lung volume segmentation, the reduced contrast from the low flip angle actually simplifies the segmentation task.

\section{Image Reconstruction}

All algorithms were implemented in Matlab (Mathworks, Natick, MA, USA). Dynamic 3D images were reconstructed from highly undersampled data using a nonlinear conjugate gradient algorithm with CS sparse penalty terms (17). Sparsity is enforced by TV regularization across both spatial and temporal dimensions.

The spatial TV of an image $u$ (with pixels indexed by $x, y, z$, and $t$ ) is defined in Eqs. [1] to [3], and is equivalent to the $\ell_{1}$ norm of a finite difference (edge map) of the image:

$$
\begin{aligned}
T V_{x}(u) & =\sum_{x, y, z, t}|u(x, y, z, t)-u(x+1, y, z, t)|, \\
T V_{y}(u) & =\sum_{x, y, z, t}|u(x, y, z, t)-u(x, y+1, z, t)|, \\
T V_{\text {spat }}(u) & =T V_{x}(u)+T V_{y}(u) .
\end{aligned}
$$

Spatial TV may also include a term in the slice direction $(z)$. We chose not to include the slice direction because the resolution is coarser $(10 \mathrm{~mm})$, and the algorithm can then be parallelized over the $z$-index.

The temporal TV of an image mirrors the spatial TV. We distinguish the two as separate TV transformations, as they lead to different amount of sparsity, and will require different regularization weights within the reconstruction optimization. The temporal TV is defined as

$$
T V_{t e m p}(u)=\sum_{x, y, z, t}|u(x, y, z, t)-u(x, y, z, t+1)|
$$


As a result of the imaging experiment, we have data corresponding to two static lung phases: the initial (maximum inspiration) and final (forced end-expiration) bookends. These two datasets are almost fully sampled, and thus lead to detailed lung images that are nearly free of artifacts from undersampling. These well resolved images are used in a third TV-like sparse transform that we call the bookend TV, defined as

$$
T V_{\text {ref }}(u)=\sum_{x, y, z, t} w_{0}(t)|u(x, y, z, 0)-u(x, y, z, t)|+w_{T}(t)|u(x, y, z, T)-u(x, y, z, t)| .
$$

This transform penalizes the reconstructed image $u$ for differing from the fully sampled bookend reference images - $u(x, y, z, 0)$ and $u(x, y, z, T)$, the initial and final bookends respectively. Constraints based on prior images have been shown to be beneficial for CT image reconstruction (18). Here, the reference image bookends are acquired together with the dynamic phase.

The bookend TV contains two functions $w_{0}$ and $w_{T}$ that weigh each frame based on their temporal distance from the initial and final bookends respectively. These functions are flexible to be set with or without prior knowledge. For example, $w_{T}(t)$ may be set based on the raw data magnitude during the dynamic phase shown in Figure 1, normalized to range from 0 to 1 ; then $w_{0}(t)$ could be its mirror. Alternatively, $w_{T}(t)$ may be a simple linear function from 0 to 1 over the course of the dynamic time index, then $w_{0}(t)=\left(1-w_{T}(t)\right)$. We have found this linear weight sufficient, and used it in the results here. The sparsity between each time point and the bookends is illustrated in Figure 2.

Altogether, these regularizations are combined with a data fidelity $\left(\ell_{2}\right)$ constraint to solve for a reconstructed image $u$ based on the optimization problem

$$
\underset{u}{\operatorname{argmin}}\|F(S u)-d\|_{2}^{2}+\lambda_{1} T V_{\text {spat }}(u)+\lambda_{2} T V_{\text {temp }}(u)+\lambda_{3} T V_{\text {ref }}(u) .
$$

Here $F$ is the undersampled, 3D stack of stars Fourier transform at each time point. It is important to emphasize that $u$ denotes the image data at all time points including the bookends. $S$ transforms the underlying image to the spatially varying receiver coil images by applying auto-calibrated sensitivity maps. $d$ represents the acquired dynamic 3D multi-channel data. Each of the sparse TV regularizations has a parameter $\lambda_{i}$ controlling its weight in the optimization problem. The selection of these parameters is described below, and they were held constant over all subjects.

The sensitivity maps $S$ were produced using the method of Uecker et al. (19) called ESPIRiT. ESPIRiT is an auto-calibrating method for producing sensitivity maps based on eigenvalue decompositions of the Fourier transforms of the low frequency components of the raw data. A time averaged image containing all phases of the data (both bookend and dynamic) was reconstructed for each coil using Non-Uniform Fast Fourier Transform (20) (NUFFT); a standard FFT was then used to transform the reconstructed images back into (Cartesian) k-space and the central portion of $\mathrm{k}$-space was used for calibration. The calibration window and kernel sizes were $16 \times 16 \times 6$ and 
$6 \times 6 \times 3$, respectively $\left(k_{x} \times k_{y} \times k_{z}\right)$. Therefore our method assumes that the coil sensitivities are fixed over time. Previous studies $(21,22)$ have shown that correcting the sensitivity maps for motion leads to improved image quality. However, after testing our proposed method with maps calibrated from different time--windows, we found no apparent difference in the resulting images. Therefore, we concluded that the high amount of undersampling and relatively coarser spatial resolution make potential improvements due to the use of time-varying coil sensitivities less significant in this application.

In summary, the image reconstruction pipeline consists of the following steps:

1. Using the correlation of the $\mathrm{k}$-space center, each radial view is assigned to one of three phases (initial bookend, dynamic, and final bookend phases (as in Figure 1)).

2. The full dataset (i.e. without consideration of the dynamic nature of the data) is used to create sensitivity maps with ESPIRiT.

3. The images are reconstructed by solving the problem in Eq. [6] with a nonlinear conjugate gradient algorithm using data from all three phases.

Each iteration of step 3 was run on a computer with 64GB Memory, nVidia GTX 780 GPU, and a 4-core Intel Xeon E5-1620 3.6GHz CPU. These iterations would require $60 \mathrm{~s}$ on the CPU, so we implemented a GPU version of the NUFFT (23) which helped to reduce this time to approximately $15 \mathrm{~s}$. The reconstruction ran for 150 iterations, i.e., approximately $37 \mathrm{~min}$, for each dynamic 3D image. Our Matlab code was not optimized, and this reconstruction time could be reduced by careful optimization of the code and parallelization over additional computing resources.

For comparison to the complete CS problem in Eq. [6], alternative reconstruction methods were used. One is a non-iterative method using a simple zero-padded pseudo-inverse of the NUFFT. Another, referred to as CG-SENSE (24), is a parallel imaging reconstruction method equivalent to solving Eq. [6] with $\lambda_{i}=0$.

\section{Selection of $\lambda_{i}$}

If the CS parameters are too small, the image will contain aliasing artifacts, and if they are too large, the image will be blurry. One approach to choosing these parameters could be to compare the undersampled image to a ground truth image, and calculate the root-mean-squared error or structural similarity between the two over a range of parameter values (25). However, the choice is particularly challenging in applications where a ground truth image is not available.

An alternative method (26) was developed to automatically select one parameter for an iterative soft thresholding CS MRI reconstruction, with no need for a reference image. However, this method is only suitable for "wavelet-like" sparsity bases and does not directly extend to the three-parameter nonlinear conjugate gradient method used here.

In this work, the regularization parameters were determined with the goal of achieving a clear 
definition of the lung-chest-diaphragm boundary. It was empirically determined that the parameters $\lambda_{1}=5 \times 10^{-5}, \lambda_{2}=10 \times 10^{-5}$, and $\lambda_{3}=5 \times 10^{-5}$ provided good boundary definition across all subjects. Therefore, these fixed values were used for all subjects in this study. As was noted by Peng et al. (27), "the selection of regularization parameters with at least one or two penalties is still an open problem in the field of optimizations." Our proposed reconstruction method stands to be improved by new parameter selection techniques.

\section{Image segmentation}

The lung boundaries were segmented from the reconstructed image in order to compute lung volume. Segmentation of the lung-chest-diaphragm boundary was performed manually using MRIcro (C. Rorden, freeware). The segmented lung voxels are used to track the change in lung volume over time.

\section{Conventional Spirometry}

For the purpose of comparison, MRI data were acquired simultaneously with spirometer data. Study subjects were placed supine in the MRI as per usual imaging protocol. Concurrently, subjects had a nasal clamp and a spirometry mouthpiece applied. The mouthpiece was attached to a portable spirometer (ndd Medical Technologies, Andover, MA, USA), placed remotely from the scanner, using 4 meter length, $2 \mathrm{~cm}$ inner diameter, corrugated tubing and airtight/ connectors.

\section{Results}

The resulting images capture the dynamics of the lungs in 3D during forced expiration. Figure 3 illustrates dynamic 3D segmentation of the lungs, and the changes of the volume during the dynamic phase of the breathing experiment. Supporting Video S1 demonstrates the changes of the dynamic 3D segmented lung volume in real time.

The full reconstruction optimization problem relies on various forms of regularization in both time and space to reconstruct the highly undersampled dynamics images. Figure 4 shows slices of the dynamic 3D image reconstruction $u$ that result from various forms of regularization. Each image has $156 \mathrm{~ms}$ temporal resolution based on only 3 readouts per slice per time point. In Figure 4(a), the non-iterative pseudo-inverse reconstruction was used, and it is clear just how few data are associated with each time point. Figure 4(b) shows the CG-SENSE reconstruction. An additional spatial sparse penalty is applied in Figure 4(c), which has the effect of smoothing the CG-SENSE image, but the lungs are still unresolved. We can see the drastic improvement with temporal CS in Figure 4(d). Further improvement is obtained from the bookend reference regularization in Figure 4(e). 
The images in Figure 4 use an acceleration factor of approximately 50 relative to the Nyquist criterion of an isolated time point image. Figure 5 shows four images each with differing temporal resolution, from 4 views per slice per time point down to as low as 1 view per slice per time point. The slice shown corresponds to the midpoint (or half-maximum volume) acquired during active forced expiration. There is a direct tradeoff between spatial and temporal resolution: as the number of views per time point increases, the number of time points decreases. Each image will be resolved from more complete data, but there will be fewer images and lower effective temporal resolution. In terms of Eq. [6], a change in the number of views per time point, will affect the dimensionality of the image $u$ (higher or lower dimensionality for fewer or greater views per time point respectively) but the data $d$ is unchanged. A change in the number of views will require an adjustment of the NUFFT $F$ for the k-space trajectories.

In vivo validation comes from correlation between lung volume calculations based on segmentation of the reconstructed images, and simultaneously acquired spirometer measurements. The number of voxels within the lungs decreases as the lungs expire. By tracking the change in lung voxels, and multiplying by the voxel size, the expired lung volume over time can be computed. A direct comparison of the change in volume is shown in Figure 6. The segmented MRI volume and the spirometer volume exhibit consistent dynamic characteristics for six different subjects. One thing to note is that the spirometer quantifies a different volume than the image, and that leads to a higher volume from the segmentation. This difference in measured physiological parameters is expected, and is discussed further below.

\section{Discussion}

We have demonstrated a method for volumetric imaging of the lungs during forced expiration. Current standards for PFTs measure only global changes using spirometry. By imaging during the PFT maneuvers, the observation of local lung biomechanics, such as contrasting left and right lung dynamics, or monitoring contraction of the chest and diaphragm may be possible.

The data were acquired with a radial stack-of-stars technique. In comparison to Cartesian sampling, the radial pattern offers two benefits. Firstly, the radial trajectory repeatedly measures the $\mathrm{k}$-space origin. Because the data at the origin is equal to the integral of the object over the FOV, these repeated measurements can be used to monitor the global changes in the object-here, a trace of the respiration. Secondly, the radial sampling is dense in the center of $\mathrm{k}$-space in comparison to a Cartesian pattern, making it more compatible for undersampled imaging.

The dynamic phase of the data was so extremely undersampled that a combination of parallel imaging and CS was critical for image reconstruction. Images were recovered from as few as one single line of k-space data per slice per frame as shown in Figure 6, an acceleration factor of 153 per frame. Sparse regularization utilizes redundancies within the image in order to make such 
acceleration possible. Most of this redundancy is found between temporal frames, as shown in Figure 5. Additional details can be resolved in the dynamic images from using the fully sampled bookend images as a prior image regularization as well.

In this work we compare volumes obtained from the dynamic 3D images to those measured with spirometry. As mentioned above, the difference between the curves in Figure 6 is due to differences in the measured quantities. The spirometer only measures the amount of gas expired through the mouth and is a measure of gas volume displaced from both lungs during expiration. Image segmentation measures the volume of lung-voxels lost between frames and will measure both the change in gas volume plus lung tissue volume. Armstrong et al. (28) have shown that the ratio of $\mathrm{ml}$ lung tissue per $\mathrm{ml}$ lung tissue and gas is $0.19 \pm 0.03$, and further variation is expected in the supine position due to an increase in pulmonary blood volume. Forced expiration predictably leads to increased intra-thoracic pressure, leading to a number of changes that includes vascular compression and blood pool displacement, decreased venous return and overall decreased blood volume.

Our results demonstrate MR imaging capability for the breathing maneuver of the most common, but demanding, PFT parameter: $\mathrm{FEV}_{1}$. Imaging during easier PFT maneuvers or free breathing may utilize this method as well, and should have improved spatial resolution due to lower temporal resolution requirements.

The dynamic 3D lung images are an improvement over previous MRI methods in terms of temporal resolution and lung coverage, and the volumetric segmentation is an improvement over spirometry in that it provides spatial information. However, limitations of our study include that the images only enable assessment of the lung perimeter contours; structures, such as small pulmonary blood vessels, are not visualized. This represents an opportunity for further technical development, perhaps by incorporating a contrast agent or hyperpolarized gas. Another limitation is that the validation provided here is global rather than regional, and additional validation may be required as regional applications are explored.

Future work will involve motion analysis of the dynamic 3D segmented image in order to quantitate local stress within the boundaries of the lungs during expiration. A comparison between healthy subjects and subjects with various types and stages of pulmonary pathologies will be valuable.

\section{Conclusion}

A method for volumetric imaging of the lungs during forced expiration is demonstrated. The method consists of a rapid data acquisition strategy and a complimentary image reconstruction method for obtaining dynamic 3D images from highly undersampled measurements. The acquisition strategy is flexible and allows retrospective trade-off between spatial and temporal resolutions. The reconstructed images contain the full lung volume and exhibit dynamics which correlate well with 
simultaneous spirometer measurements. The proposed method may lead to further regional analysis of lung dynamics which could serve as a biomarker for physiological pulmonary pathology.

Figures

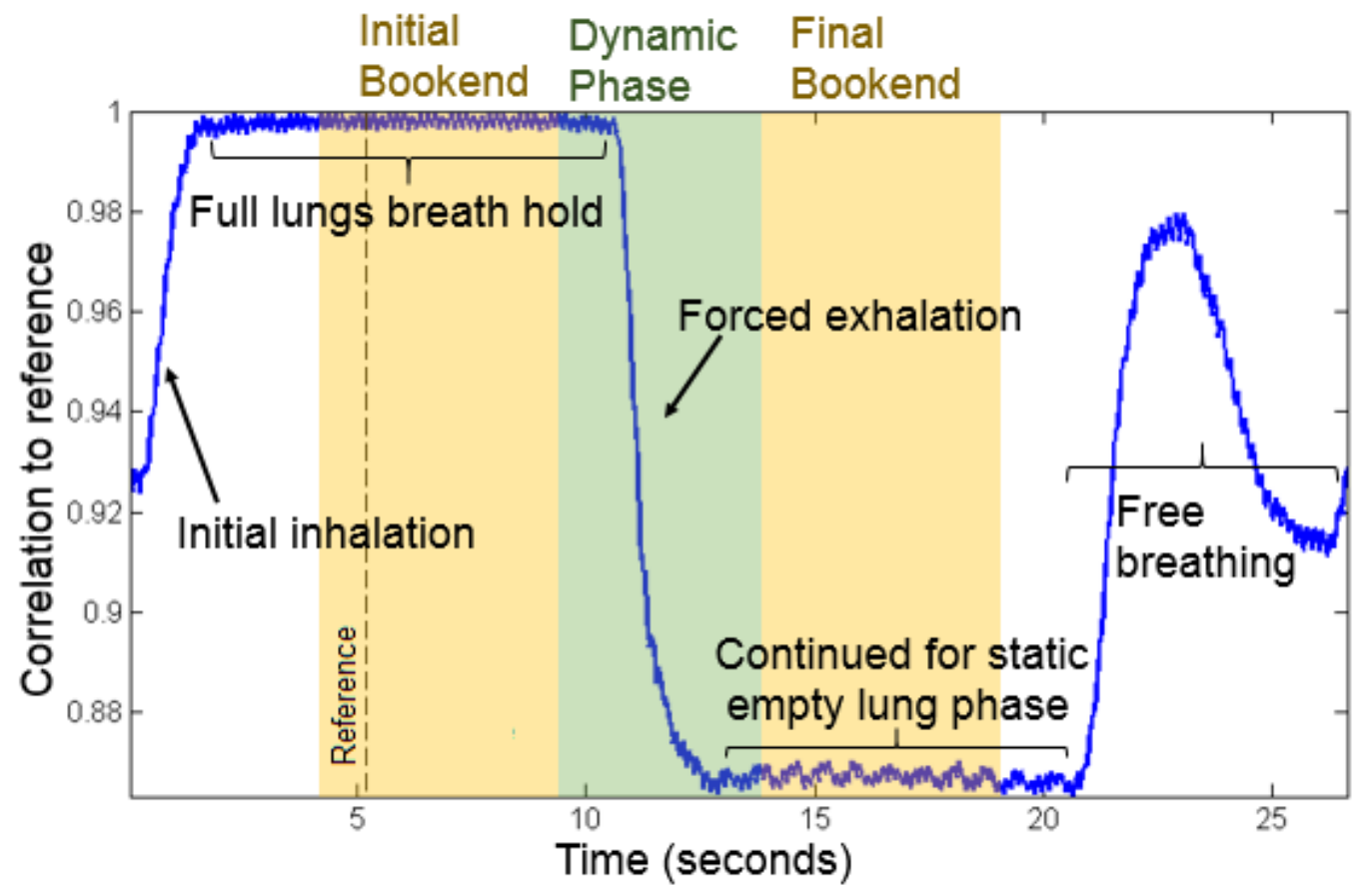

Figure 1: The correlation between the data along the center $k_{z}$ line and the reference is plotted for each angle on which it is sampled. From this raw data, the phases of the forced expiration experiment can be observed and the bins can be distributed across the dynamic phase, thus determining the temporal resolution. The initial and final bookends are placed during the static lung phases and may be fully sampled as long as the breath holds are long enough. The total time of acquisition (512 readouts per slice) is 26.7 seconds. 


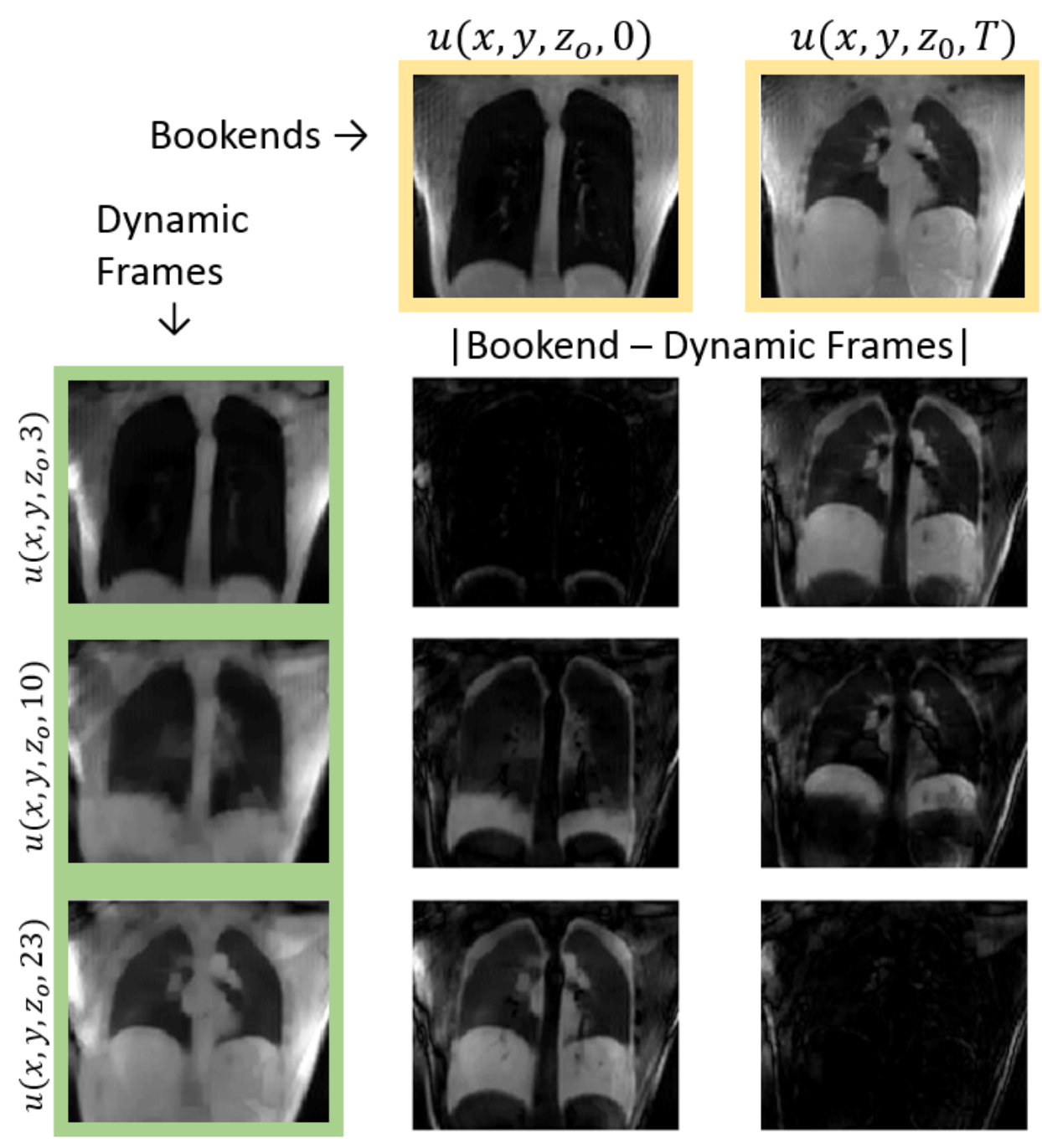

Figure 2: Absolute differences between dynamic time points (3, 10, and 23) and the bookend reference frames ( 0 and $T=26)$ from the dynamic $3 \mathrm{D}$ reconstructed lung image for selected central slice $z_{0}$. In the first column, slices of the three dynamic time points are shown. In the second column, at the top is the initial bookend; the following rows contain the magnitude of the differences with this bookend. In the third column, at the top is the final bookend, and the following rows contain the magnitude of the differences with this bookend. 

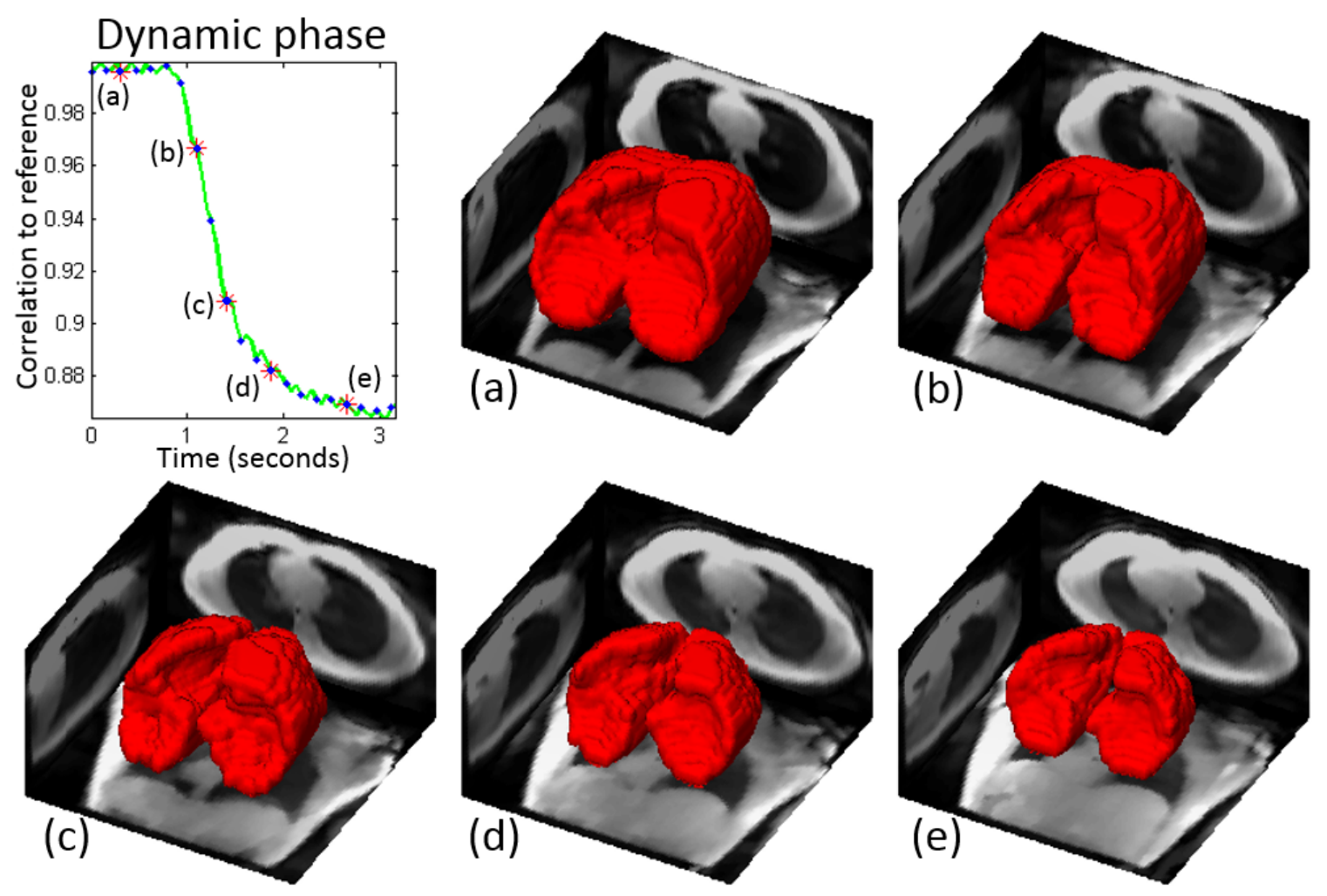

Figure 3: The top left plot shows the dynamic phase from Figure 1 - the correlation of the data along the center $k_{z}$-line to the reference (same as previous, during initial bookend). Images were reconstructed at each equally spaced blue point along the curve, and images are shown here for each red star along the curve (a) through (e). 


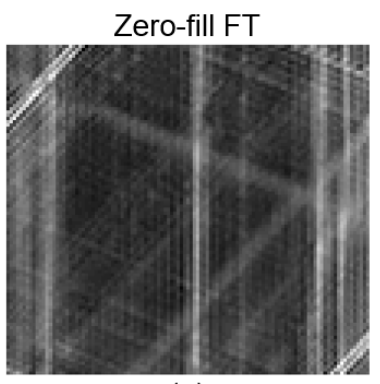

(a)

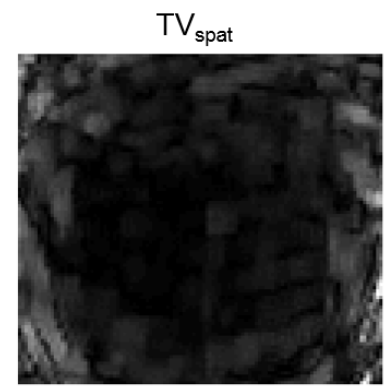

(c)

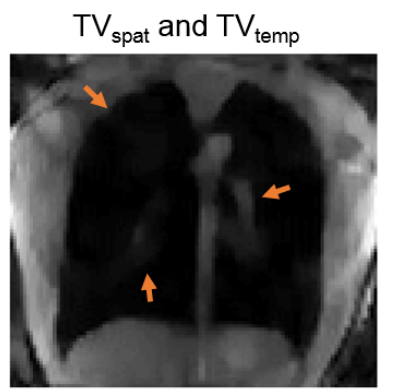

(d)

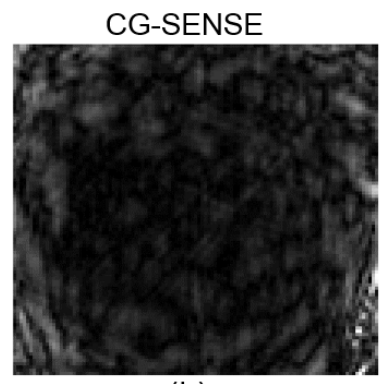

(b)

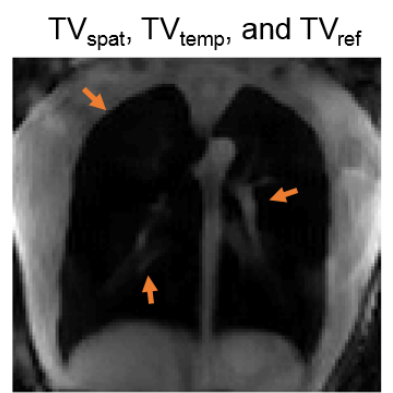

(e)

Figure 4: A selected slice and time point $(z=13, t=3)$ from a dynamic 3D reconstruction. (a) Zero-padded inverse NUFFT reconstruction, non-iterative. (b) CG-SENSE, no compressed sensing. (c) Compressed sensing with CG-SENSE and a spatial total variation penalty only. (d) Compressed sensing with CG-SENSE and spatial and temporal total variation penalties. (e) Complete compressed sensing (Eq. [6]) with CG-SENSE reconstruction with spatial and temporal total variation penalties, as well as bookend residual sparsity. Arrows indicate regions of improvement between (d) and (e). 


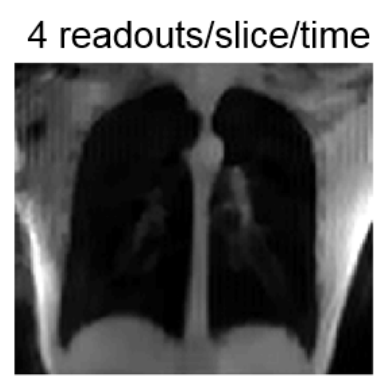

(a)

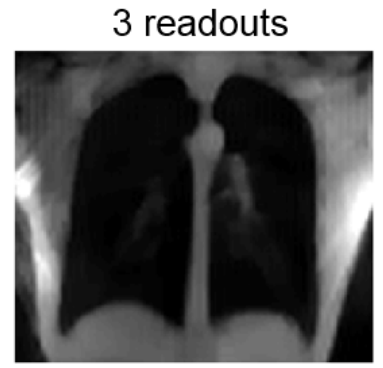

(b)

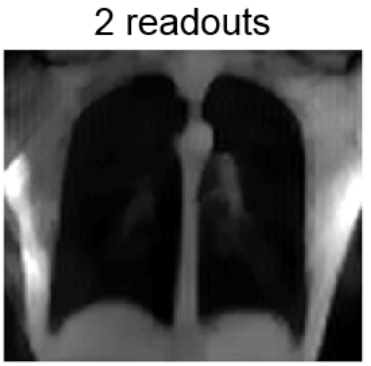

(c)

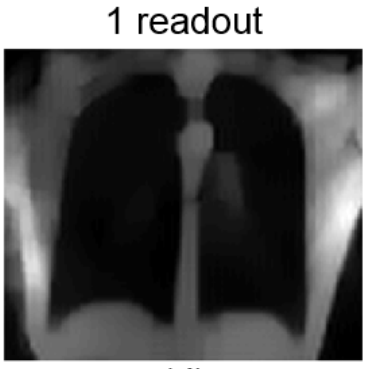

(d)

Figure 5: The spatial-temporal resolution can be selected retrospectively by including different amounts of data per time point. Here are example images of a selected slice and time point from the middle of the dynamic phase, with (a) 4 views per slice per time, 104 views per time, acceleration factor 38, 208 ms resolution, (b) 3 views per slice per time, 78 views per time, acceleration factor 51, $156 \mathrm{~ms}$ resolution, (c) 2 views per slice per time, 52 views per time, acceleration factor 76, $104 \mathrm{~ms}$ resolution, (d) 1 view per slice per time, 26 views per time, acceleration factor 153, 52 ms resolution. 

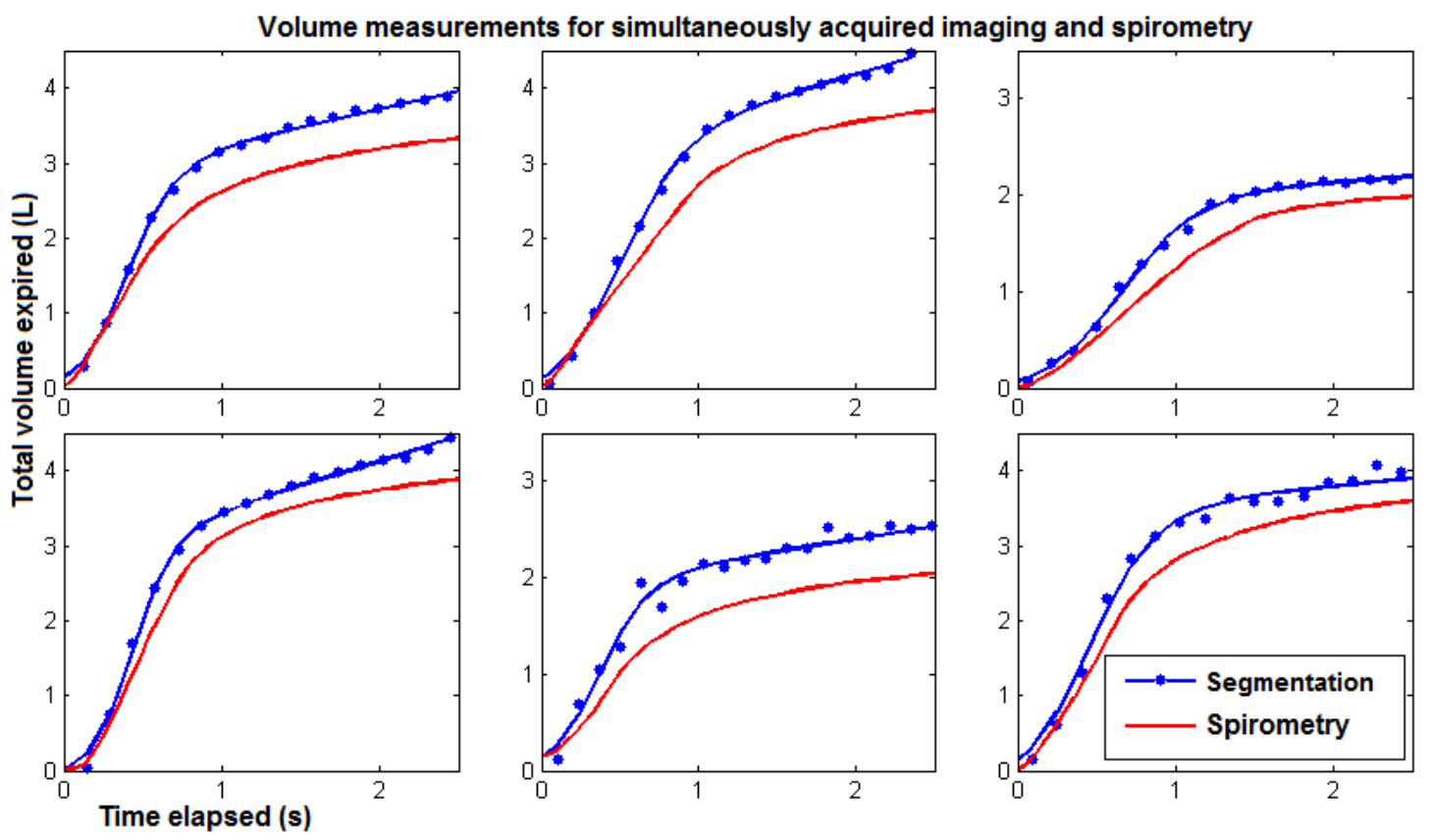

Figure 6: A comparison of expired volume over time between image segmentation and spirometer measurement for six healthy subjects. The blue points represent segmentation, and the red curve represents the spirometer measurement. For display purpose only, the segmentation data were fit to a modified logistic curve, shown in blue. Each corresponding image was reconstructed with 3 views per slice per time. Segmentation measures the change in volume of the air plus the tissue within the lungs. Spirometer measures the change in volume of just the air that is expired through the mouth.

\section{Supporting Information}

https://youtu.be/V24rjxUz1Jo

Supplemental Video S1. The motion of the lungs during forced expiration is shown in real time (132 ms frames) for the full segmented volume, as in Figure 3. Three radial views per slice per time point were used for the reconstruction. 


\section{Acknowledgement}

Benjamin Paul Berman is grateful to have received funding from the Phoenix Chapter of the ARCS Foundation.

\section{References}

1. Takahashi M, Fukuoka J, Nitta N, Takazakura R, Nagatani Y, Murakami Y, Otani H, Murata K. Imaging of pulmonary emphysema: a pictorial review. Int J Chronic Obstr Pulm Dis 2008;3(2):193-204.

2. Gibson G. Pulmonary hyperinflation a clinical overview. Eur Respir J 1996;9(12):2640-9.

3. McKusick KA, Wagner Jr. H, Soin JS, Benjamin JJ, Cooper M, Ball Jr. WC. Measurement of regional lung function in the early detection of chronic obstructive pulmonary disease. Scand J Respir Dis Suppl 1974;85:51.

4. Chen Q, Mai VM, Bankier AA, Napadow VJ, Gilbert RJ, Edelman RR. Ultrafast MR gridtagging sequence for assesment of local mechanical properties of the lungs. Magn Reson Med 2001;45(1):24-8.

5. Voorhees A, An J, Berger KI, Goldring RM, Chen Q. Magnetic resonance imaging-based spirometry for regional assessment of pulmonary function. Magn Reson Med 2005;54(5):1146-54.

6. Sundaram TA, Gee JC. Towards a model of lung biomechanics: pulmonary kinetics via registration of serial lung images. Med Imag Anal 2005;9(6):524-37.

7. Zhang K, Voorhees A, Grossman E, Berger KI, Goldring RM, Reibman J, Xu C, Dougherty L, Chen Q. Assessing local lung function: measurement of regional FEV1/FVC using tissue tracking MRI. In the Proceedings of the 16th Annual Meeting of ISMRM, Toronto, Ontario, Canada, 2008. p. 3787.

8. Möller HE, Chen XJ, Saam B, Hagspiel K, Johnson GA, Altes TA, de Lange EE, Kauczor HU. MRI of the lungs using hyperpolarized noble gases. Magn Reson Med 2002;47(6)1029-51.

9. Eichinger M, Tetzlaff R, Puderbach M, Woodhouse N, Kauczor HU. Proton magnetic resonance imaging for assessment of lung function and respiratory dynamics. Eur J Radiol 2007;64(3):32934 .

10. Plathow C, Ley S, Fink C, Puderbach M, Heilmann M, Zuna I, Kauczor HU. Evaluation of chest motion and volumetry during the breathing cyclce by dynamic MRI in healthy subjects: comparison with pulmonary function tests. Investig Radiol 2004;39(4):202-9. 
11. Clausen J. Measurement of absolute lung volumes by imaging techniques. Eur Respir J $1997 ; 10(10): 2427-31$.

12. Tokuda J, Schmitt M, Sun Y, Patz S, Mountford CE, Hata N, Wald LL, Hatabu H. Lung motion and volume measurement by dynamic 3D MRI using 128-channel receiver coil. Acad Radiol 2009;16(1)22-7.

13. Ajraoui S, Lee KJ, Deppe MH, Parnell SR, Parra-Robles J, Wild JM. Compressed sensing in hyperpolarized 3He lung MRI. Magn Reson Med 2010;63(4)1059-69.

14. Lingala SG, Baqqal Y, Newell J, Wang D, Sieren J, Thedens D, Jacob M. Towards 3D dynamic MRI of the lung using blind compressed sensing. In the Proceedings of the 22nd Annual Meeting of ISMRM, Milan, Italy, 2014. p. 0298.

15. Feng L, Grimm R, Block KT, Chandarana H, Kim S, Xu J, Axel L, Sodickson DK, Otazo R. Golden-angle radial sparse parallel MRI: combination of compressed sensing, parallel imaging, and golden-angle radial sampling for fast and flexible dynamic volumetric MRI. Magn Reson Med 2014;72(3):707-17.

16. Winkelmann S, Schaeffter T, Koehler T, Eggers H, Doessel O. An optimal radial profile order based on the golden angle ratio for time-resolved MRI. IEEE Trans Med Imaging 2007;26(4):6876.

17. Lustig M, Donoho D, Pauly JM. Sparse MRI: the application of compressed sensing for rapid MR imaging. Magn Reson Med 2007;58(6)1182-95.

18. Chen GH, Tang J, Leng S. Prior image constrained compressed sensing (PICCS): a method to accurately reconstruct dynamic CT images from highly undersampled projection datasets. Med Phys 2008;35(2)660-3.

19. Uecker M, Lai P, Murphy MJ, Virtue P, Elad M, Pauly JM, Vasanawala SS, Lustig M. ESPIRiT - an eigenvalue approach to autocalibrating parallel MRI: where SENSE meets GRAPPA. Magn Reson Med 2014;71(3):990-1001.

20. Fessler JA, Sutton BP. Nonuniform fast Fourier transforms using min-max interpolation. IEEE Trans Signal Process 2003;51(2):560-74.

21. Schmidt JF, Boesiger P, Kozerke S. On moving coil encoding and calibration in dynamic imaging. In the Proceedings of the 20th Annual Meeting of ISMRM, Melbourne, Victoria, Australia, 2012. p. 2464.

22. Bammer R, Aksoy M, Liu C. Augmented generalized SENSE reconstruction to correct for rigid body motion. Magn Reson Med 2007;57(1):90-102. 
23. Hansen MS, Sørensen TS. Gadgetron: An open source framework for medical image reconstruction. Magn Reson Med 2013;69(6):1768-76.

24. Preussmann KP, Weiger M, Bornert P, Boesiger P. Advances in sensitivity encoding with arbitrary k-space trajectories. Magn Reson Med 2001;46(4):638-51.

25. Hollingsworth KG, Higgings DM, McCallum M, Ward L, Coombs A, Straub V. Investigating the quantitative fidelity of prospectively undersampled chemical shift imaging in muscular dystrophy with compressed sensing and parallel imaging reconstruction. Magn Reson Med 2014;72(6):16109 .

26. Khare K, Hardy CJ, King KF, Turski PA, Marinelli L. Accelerated MR imaging using compressed sensing with no free parameters. Magn Reson Med 2012;68(5):1450-7.

27. Peng X, Ying L, Liu Q, Zhu Y, Liu Y, Qu X, Liu X, Zheng H, Liang D. Incorporating reference in parallel imaging and compressed sensing. Magn Reson Med 2015;73(4):1490-1504.

28. Armstrong JD, Gluck EH, Crapo RO, Jones HA, Hughes JMB. Lung tissue volume estimated by simultaneous radiographic and helium dilution methods. Thorax 1982;37(9):676-9.

29. Berman BP, Pandey A, Li Z, Trouard T, Oliva I, Martin DR, Altbach M, Bilgin A. Very rapid 4D compressed sensing MR for lung imaging during forced expiration. In the Proceedings of the 22nd Annual Meeting of ISMRM, Milan, Italy, 2014. p. 4367. 